DOI: http://dx.doi.org/10.33846/hn50206

http://heanoti.com/index.php/hn

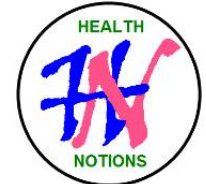

CASE REPORT ARTICLE

URL of this article: http://heanoti.com/index.php/hn/article/view/hn50206

\title{
Consultation Liaison Psychiatry on Case Management of Children with Mixed Gonadal Dysgenesis Mosaicism 45X (60\%) and 46XY (40\%)
}

\begin{tabular}{c} 
Jeffrey Agung ${ }^{1(C A)}$, Yunias Setiawati \\
${ }^{2}$ \\
${ }^{1(\mathrm{CA})}$ Division of Child and Adolescent Psychiatry, Department of Psychiatry, Faculty of Medicine, Universitas \\
Airlangga / Dr. Soetomo Hospital, Indonesia; jeffry15129@ gmail.com (Corresponding Author) \\
2Division of Child and Adolescent Psychiatry, Department of Psychiatry, Faculty of Medicine, Universitas \\
Airlangga / Dr. Soetomo Hospital, Indonesia; yunias.setiawati@gmail.com \\
\hline
\end{tabular}

\section{ABSTRACT}

There is an increasing in admitted patients with cases of gender identity disorder in the daycare clinic of the Child and Adolescent Psychiatry Division Dr. Soetomo, it is necessary to increase understanding and clinical ability in relation to gender identity disorders through psychiatry consultation discussion, so that management can be achieved properly and comprehensively. Disorder of sex development (DSD) is a medical disorder that is associated with an incompatibility between chromosomes, gonads, phenotypes and anatomy which is characterized by the development of external genital organs that are incompatible with whether it is male or female. Genetalia ambiguos can cause significant psychological stressors for patients or their families, where as a result of the conditions experienced, it will lead to gender dysphoria with the resulting effects, among others, in the form of anxiety, depression and low self-esteem, to affect the quality of life of children as adolescents and adults. . The method of preparing this report is a case analysis and the role of CLP which is given based on the results of the examination and references to management in the field of psychiatry of children and adolescents in cases of DSD from children aged 12 years. Conducted to strengthen the adaptation of patients to problems identifying gender development and to their families by emphasizing the importance of achieving optimal quality of life for children. The results of the case reports describe the role of Consultation Liason Psychiatry through communication and consultation between related areas of expertise, supportive psychotherapy and continuous Cognitive Behavior Therapy (CBT) techniques along with psychoeducation in families providing good development results with results in reducing patient anxiety and depression, based on children's scoring Depression Inventory (CDI) of patients with Mosaicism, Mixed Gonadal Dysgenesis (MGD). The conclusion of this case report is that the problem of psychological stressors that arise in DSD requires psychiatric assistance according to the stages of child development. The important role of psychiatrists in the management of DSD cases has been recognized and stated in the joint consensus for the treatment of individuals with DSD since 2006. CLP needs to be built because of the complexity of treatment and therapy in DSD cases, which involve subspecialty pediatric endocrinology, urology, psychiatry, gynecology, genetics, workers. social nurse and medical ethics. described in the algorithm below in the management of cases of gender dysphoric.

Keywords: disorder of sex development; mixed gonadal dysgenesis; consulation liason psychiatry disorder of sex development; mixed gonadal dysgenesis; consulation liason psychiatry

\section{Background}

\section{INTRODUCTION}

The incidence of DSD occurs in 1: 4,500 to 1: 5,500 births, a state of development of the male or female genital organs that is different from normal. Abnormal conditions in the development of sex chromosomes, gonads, or the anatomy of the genital organs. The disruption in the process of forming the genital organs causes imperfection and function of the genital organs. The term DSD is used to replace old terms that cause discomfort to families and patients such as intersex, ambiguous, pseudohermaphrodite, hermaphrodite, and sex reversal ${ }^{(1)}$. Disorders of the development of the genital organs can be caused by several factors, namely: (1) genetic factors that determine the formation of gonads. (2) gonad factors that determine which hormones will work, (3) hormonal factors that determine the phenotype (internal and external genitalia) that will be formed. DSD can be defined as a condition characterized by: 1. congenital confusion of genital organs (46xx virilization 
of $\mathrm{CAH}$, cliteromegaly, micropenis), 2. congenital disorders of separation of external and internal genital anatomy (AIS, Def 5Alfa reductase), 3. development of genital anatomy incomplete (vaginal agenesis, gonadal egenesis), 4. sex chromosomal anomalies (Turner syndrome, Kleneifelter syndrome, mosaicism sex chromosomes), 5. gonadal development disorders (ovo-testis) ${ }^{(2)}$. Mixed gonadal dysgenesis is characterized by the presence of a line gonad (streaky) and an imperfect testis where patients with genetic Mosaicism with karyotypes $45, \mathrm{X}$ and $46, \mathrm{XY}$ with multiple genitalia is a variant of the chromosomal abnormality found in turner syndrome disorder where the picture of karyotype there is element $\mathrm{X}$ incomplete in the cell line in the genetic process. It said the incidence of Mosaicism was 1: 2000 births. MGD is the second most ambiguous group after $\mathrm{CAH}^{(3)}$.

Psychosexual development is influenced by three main factors, namely gender identity, gender roles and gender orientation. Since the last few years, understanding of the development of gender identity disorders has grown rapidly since the development of genetic examination and imaging technology, so that it can help efforts to enforce and manage DSD. The existence of a mismatch between chromosomes, gonadal development and phenotypes can cause psychosexual problems, especially in pediatric patients, where the importance of gender identity is involved in determining and shaping the patient's personality and behavior in the phase of mental development. Therefore, the management of DSD patients must follow the principles of patient and family centered services where the management given really pays attention to aspects of the patient's needs and minimizes the psychological burden and his family ${ }^{(4)}$. This paper discusses cases of anxiety disorders, depression and low self esteem in children with MGD, Mosaicism 45X (60\%) and 46XY (40\%) and their management.

\section{Purpose}

The case report on sentence consultation psychiatry on child case management with mixed gonadal dysgenesis Mosaicism aims to strengthen patient adaptation to the problem of identifying gender development and towards their families by emphasizing the importance of achieving optimal quality of life for children.

\section{METHODS}

The method of writing this article is a case report on sentence consultation liason psychiatry on the management of cases of children with mixed gonadal dysgenesis Mosaicism. The aim is to strengthen patient adaptation to the problem of identifying gender development and to their families by emphasizing the importance of achieving optimal quality of life for children. Using a case analysis approach and the role of CLP that is given based on the results of the examination and references to management in the field of child and adolescent psychiatry in DSD cases from children aged 12 years. The case reported was one child aged 12 with a DSD case. The location for taking cases in the daycare clinic of the Child and Adolescent Psychiatry Division Dr. Soetomo. The observation time was carried out for two months. Analysis of the results of the case reports is conducted descriptively based on the findings through the observation process.

\section{RESULTS}

This case report discusses cases of anxiety disorders, depression and low self-esteem in children with MGD, Mosaicism 45X (60\%) and 46XY (40\%). The results of the case illustration from this case report are Ms. M, 12 years old, came with both parents to the Child and Adolescent Psychiatry clinic of Dr. Soetomo Hospital Surabaya, a referral from the obstetrics and gynecology section with requests for management assistance related to examination findings from the gynecology and gynecology fields. and urology, namely vaginal agenesis, pro surgery.

\section{General Examination Results}

Physical examination of the general condition was normal, there was no abnormality in generalist status, on gynecological examination of the labia major $(+)$ and the left side, there was a mass with a size of 2 $\mathrm{cm}$, labia minor (+), clitoris (-), OUE (+), vaginal introitus (+).

The results of the ultrasound examination were that there was no visible picture of the uterus, you could see a testicle size of $\pm 1.6 \times 0.8 \times 2.1 \mathrm{~cm}$ in the right inguinal region, a testicle of $\pm 2.1 \times 0.9 \times 1.2 \mathrm{~cm}$ was seen in the left labia, liver / GB / spleen / pancreas. / kidney no abnormality.

The results of the IVP CT examination were obtained; Prostate formation in the posterior bladder measuring $\pm 1.0 \times 0.85 \times 0.4 \mathrm{~cm}$, no clear appearance of the uterus, visible testes in the formations such as the left scrotum and undecensus of the right testis in the right inguinal.

The results of the genitographic examination showed that the hole at the bottom of the vaginal formation with a picture of the urethra associated with the bladder, did not show the structure of the mullerian duct.

The results of karyotyping obtained results from 100 cells analyzed, obtained Mosaicism, namely karyotype 45, X (60\%) and karyotype 46, XY (40\%). 


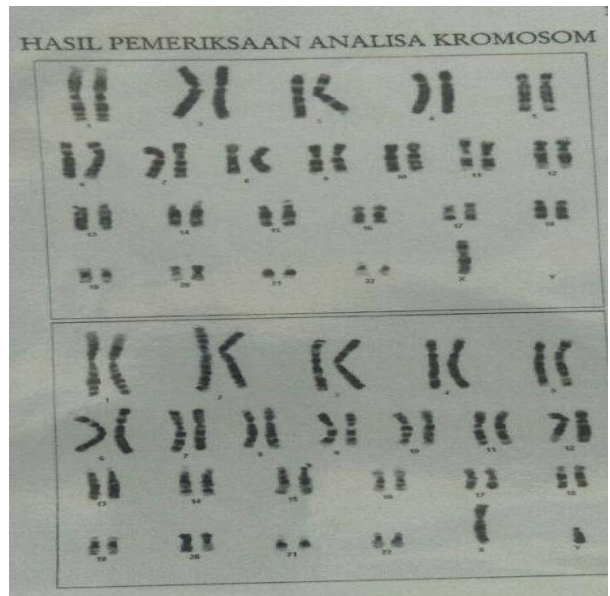

Figure 1. Results of 46X and 46XY Mosaicism karyotyping
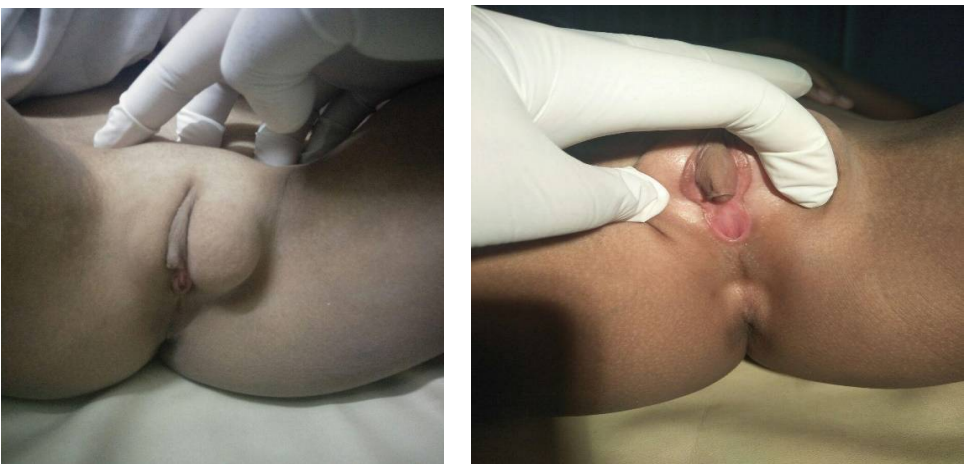

Figure 2. Results of external genetalia examination

Table 1. Hormonal test results

\begin{tabular}{ccl}
\hline & \multicolumn{2}{c}{ Hormonal examination } \\
\hline FSH & $1.77 \mathrm{mIU} / \mathrm{ml}$ & Male $1.5-11.8$ \\
\hline Testosteron & $578.80 \mathrm{ng} / \mathrm{dl}$ & Female $14-78$ \\
& & Males $241-827$ \\
\hline Estradiol & $7.013 \mathrm{pg} / \mathrm{ml}$ & Males: $<87$ \\
\hline LH & $3.049 \mathrm{mIU} / \mathrm{ml}$ & Males: $1.1-25$ \\
& & Females: \\
& & Folikular phase:1.2-12.5 \\
& & Luteal phase $0.4-19$ \\
& & Ovulatory phase $12-82$ \\
\hline
\end{tabular}

\section{Consultation Liason Psychiatry}

Urology: In this case, as clinically examination founded there is a testis, it is probably still functioning, for the testis biopsy plan is currently not recommended because it breaks the blood testicular barrier which can cause injury (becomes antisperm, perineal hypospadias typical of mixed developmental sexual disorder, undecensus testis is a possibility. 30\% malignancy occurred). Andrology: Regarding the administration of HCG, the testes if not lowered. The risk of malignancy increases, if lowered it does not reduce the number of malignancies but is easier to evaluate. Pediatric: Patients with DSD, MDG (mixed Gonadal dysgenesis) Mosaicism 99\% infertile, high FH and testosterone hormonal profile does not need special examination. In patients who can be directed either to be male or female but cannot be perfect, it is better to wait until gender maturity (age 18 years) when the patient can determine his own identity. Gynecological: Gonadectomy is performed to prevent gonadoblastoma, ideally waiting until the patient can determine himself. Issues focus on gender, sexuality, reproduction and risk of malignancy. Medicolegal considerations are needed regarding gender determination. Forensic: before action is taken it needs to be decided in court, and it needs to include the results 
of the discussion and the results of the patient and family agreement. The population law states that decisions can be made if the patient already has a identity card.

\section{Psychiatric Examination}

Autoanamnese showed that the patient wanted to immediately get treatment for the "lump" in his genital area, felt uncomfortable and embarrassed about the disorder, had been made fun of by his friend because of his small breasts, wanted to be a "woman", because of his daily interactions. day with his girlfriend and likes to cook. Previously as a child when urinating was a bit painful, now it's reduced. Heteroanamnese (mother) was born normally in a midwife, at term and immediately cried, when she was 5 years old she was hospitalized for 1 week because of typhoid, development and growth were in accordance with her age. When he was 5 years old, the patient had asked his mother about the bulge around his genitals. The urinating complaint caused the patient to be taken to the hospital. Initially it was suspected of having a "hernia", when the operation was to be carried out a "penis" was found, so the operation was canceled, the patient was referred to the top referral hospital Soetomo. Cost constraints make further action delayed. Patients are classified as obedient children who are sociable, spoiled, and have been treated as girls by their families.

Psychiatric status and supporting examinations were obtained, the patient was a child with a female appearance wearing a headscarf, quite calm and able to respond to interviews well, awareness of compos mentis, adequate mood / affect, good eye contact, smooth relevant verbal contact, preoccupation thinking process on genital problems, good understanding. Initial CDI score of 15. IQ 100-105, enough to be able to analyze even though it is simple, so that it is able to understand things that require deeper thinking. Socially, patients are able to involve themselves in the social environment, although they tend to be careful and lack self-confidence, so that when faced with new situations, they seem insecure. Emotionally enough to be able to show and control herself well, so as to be able to manage her emotions positively, have a firm stand, so that she is able to actualize herself as expected, especially as a girl.

Multiaxial diagnoses which are Axis 1: Sexual Identity Disorders (F.64); Axis 2 has not yet formed a personality; Axis 3: MGD and Mosaicism; Axis 4: problems with patient perceptions and parental expectations; Axis 5: GAF current scale 70-60, last 1 year 90-80

\section{Management}

Psychopharmaceutical therapy is postponed unless in the course of management there are significant signs and symptoms that lead to anxiety disorders and / or depression, so that psychopharmaceutical administration is reconsidered.

Non-psychopharmaceutical therapy includes; (1) supportive psychotherapy with reassurance and reinforcement or strengthening the patient's ability to adapt to both internal and external stressors in connection with her own problems and developmental problems both as women and men and their suitability for patients. (2) Cognitive behavioral therapy (CBT) aims to identify negative thoughts or cognitive distortions of the patient towards problems that make the patient feel uncomfortable or tend to be anxious in the long-term management journey. (3) Family psychoeducation: providing understanding and knowledge of the patient's actual condition and consciously understanding the possibilities that will occur in the patient associated with the patient's strong parental desire to become a "woman". Where the results of examinations from the fields of Obstetrics, Pediatrics, Urology, Andrology and Forensics show that ambiguous sex conditions in patients can be directed at the identity of men or women, this is very dependent on the patient's decision. (4) Liaison psychiatrics: provides feedback to colleagues in the field of what has been done by the psychiatrist regarding the patient's perceptions and family expectations and provides education about further actions.

\section{DISCUSSION}

DSD or in general psychology terms is ambiguous genitalia which has a significant impact on psychological stressors for both the patient and the parents. As Psychopathological it will stimulate a psychological response system due to an imbalance of neurotransmitters in the brain, as explained in the mechanism of mental disorders by Hans Selye called General Adaption Syndrome (GAS) ${ }^{(5)}$, which is a system that automatically arises when the human body responds. to a biological change as a result of a stimulus, both internal and external to the human body, the triggering of the body's defense reaction mechanism through a molecular biological sequence, to produce reactions either to changes in autonomic function or cognitive function and mental behavior, through the interaction of the components contained in the HypothalamicPituitary Axis (HPA-axis). The GAS stages consist of: 1. the alarm stage, at this stage the nervous system is awakened and the body's defenses are mobilized; 2. the stage of resistance or adaptation, which is when the mobilization determines to "flight or fight", at this stage the body is able to overcome the dose of stressor transactions; 3. the stage of exhaustion, when stress continues, causes damage to adaptation and homeostasis mechanisms ${ }^{(6)}$. Thus it can be explained of the psychopathological to the emergence of mental disorders due to psychosocial stressors in gender dysphoric disorder ${ }^{(7)}$. 
The mental disorder that most often arises in patients with DSD is a picture of gender dysphoric disorder or the patient's discomfort with his gender. According to the DSM 5 classification, it is characterized by anxiety or depressive symptoms for approximately 6 months, the emergence of a feeling of inferiority, which affects the patient's skills in social relationships with family and friends and the environment, and if this continues it will have an impact on his daily function, mental development and quality of life of children. As children reach adulthood, they will be followed by very drastic changes in psychological and hormonal development, so it is important to do gradual psychotherapy according to the patient's mental condition so that the patient has a picture of himself in the future ${ }^{(8,9)}$.

At the age of 5 years, the patient began to notice a slightly different shape of the genital organs and tried to ask his parents, but did not get an answer that could be understood by the patient. The parents only explained that the patient was a woman and there was no significant problem with the condition of the genital organs. The patient grows up as a woman, even though he feels something disturbing his genital organs. He went through school well, although the patient himself was still surprised by the shape of the genital organs. Cost limitations make it difficult for parents to check patients. In further development, the patient begins to see the difference between himself and his friends. At the age of 12, several female friends once taunted the patient for her undeveloped "breasts". This condition is a stressor for the patient and has an impact on the emergence of anxious reactions which make the patient even more worried about the enlarged lump on his genitals. The doctor's examination revealed that the female genital organs were not growing properly, and there was a possibility that the patient was a boy. Patients are increasingly confused in their uncertainties, tend to withdraw from social relationships, are not confident, and start crying a lot because of their condition, thus starting to show signs of depression ${ }^{(9)}$.

As is the case above, a girl who is born with genitals similar to a boy makes parents very depressed and difficult to accept the unnatural conditions in this society. Parents generally feel ashamed, guilty, anxious about their children's future, depressed, so they tend to be overprotective and limit children's interactions and withdraw from social involvement. Psychoeducation with the aim of providing understanding to parents about the importance of achieving optimal quality of life for children compared to questioning the types of toys, clothes also behavior of their girls which tend to be masculine or feminine, so that gender identity assessment needs to be carried out carefully and objectively (avoiding the influence of preferences. parents to a certain gender of the child) and need to consider whether the tendency of parenting patterns to a certain gender, social acceptance / support for a certain sex in the family, in the school, and the child's appreciation of their gender identity (feeling happy or depressed living in a certain gender).

\section{CONCLUSION}

Psychological stressor problems that arise in DSD require psychiatric assistance according to the child's developmental stages. The important role of psychiatrists in the management of DSD cases has been recognized and stated in the joint consensus for the treatment of individuals with DSD since 2006. Consultation liaison psychiatric needs to be built because of the complexity of treatment and therapy in DSD cases, which involve subspecialty pediatric endocrinology, urology, psychiatry, gynecology, genetics, workers. social nurse and medical ethics. described in the algorithm below in the management of cases of gender dysphoric.

\section{REFERENCES}

1. American Psychological Association. Guidelines for psychological practice with transgender and gender non conforming people. Washington DC: American Psychological Association; 2015.

2. American Psychological Association. DSM 5 diagnostic for "gender dysphoria. Washington DC: American Psychological Association; 2016.

3. Magalhães CL, Ellen SM "gender dysphoria". In: The SAGE Encyclopedia of Psychology and Gender. 2017

4. Drescher J. Diagnoses Revisited: The Past and Future of Homosexuality and Gender Diagnoses in DSM and ICD. International Review of Psychiatry. 2015;27(5):386-395.

5. Fink FG. Stress, Definitions, Mechanisms, and Effects Outlined: Lessons from Anxiety. Parkville, VIC, Australia: Institute of Neuroscience and Mental Health, University of Melbourne; 2016

6. Indyk JA. Disorders/Differences of Sex Development (DSDs) For Primary Care: The Approach to The Infant with Ambiguous Genitalia. Review Article. Transl Pediatr. 2017;323-334. DOI: http://dx.doi.org/10.21037/tp.2017.10.03

7. Julio GF, Cristina ASJ, Jesús BC, José ABV. Management Guidelines for Disorders/Different Sex Development (DSD). An Pediatr (Barc). 2018;315.

8. Michelle LB, Sarah W. A Systematic Review of Adrenarche As A Sensitive Period in Neurobiological Development and Mental Health. Developmental Cognitive Neuroscience. 2017;25:12-28.

9. Capetillo VNC, Jalil PSI, Motilla NK. Gender dysphoria: An overview. Elsevier. Medicina Universitaria. 2015; 17:53-58. 